MKG-Chirurg 2010 · 3:98-99

DOI 10.1007/s12285-009-0138-6

Online publiziert: 5. Mai 2010

(c) Springer-Verlag 2010
J. Fedderwitz

Kassenzahnärztliche Bundesvereinigung, Köln

\section{Endodontie und chirurgische Zahnerhaltung}

\author{
Gratwanderung zwischen \\ Wirtschaftlichkeit, Qualität und Ethik
}

Die ketzerische Anmerkung gleich vorweg: Geht es heute überhaupt noch um eine Gratwanderung zwischen Endodontie und chirurgischer Zahnerhaltung? Liegen die Konfliktlinien angesichts der medizinisch-technischen Entwicklung nicht längst anders? Geht es nicht im Kern um die Alternative zwischen einer mühsamen Revision mit unsicherer Diagnose einerseits und einer scheinbar leichter zu realisierenden Implantatversorgung mit vermeintlich gesicherter Überlebenszeit andererseits? Lautet die Kernfrage nicht: Was ist „Zahnerhalt" heute wert?

Sicher ist: Die Wurzelkanalbehandlung und ihre mögliche Revision sind seit Jahrzehnten anerkannte Verfahren der Zahnerhaltung. Dabei lässt sich eine erfolgreiche endodontische Behandlung nach den Richtlinien der Europäischen Gesellschaft für Endodontologie (ESE) definieren. Die Erfolgsaussichten einer Revision sind im Vergleich zur Erstbehandlung sicher geringer; zudem ist eine Revision meist schwieriger und in der Regel aufwendiger. Die Prognose ist anerkanntermaßen bei vorbehandeltem, infiziertem Endodont deutlich schlechter als bei Zähnen, die aus behandlungstechnischen Gründen (z. B. unvollständige Wurzelfüllung) einer Revision unterzogen werden sollen.

Nun ist bei der Beratung des Patienten die Erfolgsaussicht einer Behandlung nicht das alleinige Kriterium. Es gibt eine
Vielzahl zahn- und patientenbezogener Parameter, die zu berücksichtigen sind. Für den Patienten lassen sie sich zu einer Kosten-Nutzen-Risiko-Analyse zusammenfassen, die ihm die Entscheidungsfindung erleichtern soll. Die Antworten des Behandlers sind eher weniger zufriedenstellend, wenn sie sich nur auf klinische Studien beziehen. Das allein erfüllt keinen Qualitätsanspruch. Das Abweichen der Bedingungen in der Alltagspraxis von idealen Laborbedingungen, das Können und die Erfahrung des Behandlers, die technische Ausstattung seiner Praxis, das kritische Abwägen der Vor- und Nachteile eines konservativen oder endochirurgischen Verfahrens (Lokalisation, Komplikation) bestimmen die Entscheidungsfindung. Und nicht zu vergessen: Die fachliche Herkunft und persönliche Präferenz - hier der klassische „Konservist“, da der chirurgisch routinierte Behandler - weisen manchen Weg zur Therapieentscheidung.

\section{- Wesentliches fachliches Leitkriterium für eine rein qualitative Entscheidung sind die Leitlinien zur Wurzelspitzenresektion.}

Gründe für deren Erstellung waren zum einen die Prävalenz des klinischen Problems und Häufigkeit des Eingriffs, zum anderen die Diskrepanz zwischen publizierten Erfolgsraten konservativ-endo- dontischer Maßnahmen und dem in epidemiologischen Untersuchungen dokumentierten realen Versorgungsstatus. Hinzu kamen die klinisch relevanten Komplikationen bei der Wurzelspitzenresektion, aber auch der chronischen apikalen Parodontitis überhaupt. Übergeordnetes Ziel der Leitlinien ist daher auch die Verbesserung der Versorgungsqualität für die betroffene Patientengruppe.

Doch um welchen und $\mathrm{zu}$ welchem Preis? Die gesetzliche Krankenversicherung macht es sich einfach. Ihre finanziellen Ressourcen sind knapp, ihre Leistungen müssen nach dem Sozialgesetzbuch V „ausreichend, zweckmäßig und wirtschaftlich“ sein und haben „das Maß des Notwendigen" nicht zu überschreiten.

Doch bei der Revision, die der BEMA nicht als eigenständigen Bereich „kennt“, lassen die allgemeinen Behandlungsrichtlinien damit Raum für Interpretation. Dem Zahnarzt macht die GKV es damit eher schwer. Sicher, sie gibt ihm Spielräume für seine Therapieentscheidung, aber sie bürdet ihm auch die Verantwortung für diese Entscheidung auf. Er muss - zusammen mit dem Patienten - den Entschluss für oder gegen eine endodontische Behandlung fassen und die Therapie diesseits oder jenseits des Kassen-Rubikon ansiedeln. Die Richtlinien für die Molarenendo - zugegeben für den Juristen leicht, für den Zahnarzt aber eher schwer verständlich - geben dem Behandler un- 
ter bestimmten Konstellationen die Möglichkeit, eine Revision auch jenseits der GKV unter den Bedingungen der GOZ zu erbringen. Der Praxisalltag zeigt die Gratwanderung. Führt der Zahnarzt eine Wurzelkanalbehandlung nach BEMA durch, gerät er in die klassische Ethikfalle. Das BEMA-Honorar macht eine zeitliche und apparative Maximaltherapie für die Praxis unwirtschaftlich. Sie bleibt allenfalls im Rahmen einer betrieblichen Mischkalkulation machbar. Den Behandlungsaufwand zu begrenzen, kann indes die Ergebnisqualität beeinträchtigen. Die Ethikfalle schnappt ein zweites Mal zu: Was ist, wenn eine Revision zu BEMABedingungen nicht machbar ist? Ist dann die „chirurgische Zahnerhaltung“ unter Belassen der „alten Endo“ und unter Berücksichtigung der Kosten-Nutzen-Risiko-Analyse sowie der Behandlungsleitlinie eine verantwortbare GKV-Alternative?

Problematisch ist, dass die gesetzliche Krankenversicherung an diesem Punkt kein Ventil hat, keine Brücke baut zwischen gesetzlicher Grund- und privater Zusatzleistung. In der Endodontie kennt die GKV weder Mehrkostenregelung noch Festzuschüsse. An diesem Punkt gilt es anzusetzen. Für die Zukunft braucht es eine Vergütungssystematik, die unterschiedlichen Behandlungsaufwänden Rechnung trägt und die Behandlungsdauer als entscheidenden Faktor für die Ergebnisqualität anerkennt. Dann gibt es auch eine Chance, der unseligen, von interessierter Seite immer wieder aufs Neue angezettelten öffentlichen Diskussion über die vermeintlich mangelhafte Qualität von Wurzelkanalbehandlungen die Spitze zu nehmen. Einer Diskussion, die dort ebenso dumm wie unaufrichtig ist, wo sie die Anwendung des Maximalprinzips unter Missachtung der gesetzlichen Rahmenbedingungen und des ethischen Dilemmas der Praxen zum Maßstab macht. Die platten Vorwürfe, zu viele Zähne fielen der Zange zum Opfer, die Anwürfe, ein großer Teil der Endotherapien sei fachlich schlecht, haben bereits genug Schaden angerichtet. Schaden genommen hat das Image der Zahnmedizin in Deutschland. Leidtragende sind auch die Versicherten, die massiv verunsichert werden.
Bis der Gesetzgeber das Zuzahlungsverbot in der Endodontie kippt und Mehrkosten- oder Festzuschussregelungen ermöglicht, werden noch einige dicke Bretter zu bohren sein. So lange wird der Zahnarzt seine Therapieentscheidungen weiterhin im magischen Dreieck zwischen Wirtschaftlichkeit, Qualität und Ethik fällen müssen. Ohne ethische Grundeinstellung geht es dabei nicht. Sie ist das Zünglein an der Waage, in deren einer Schale die betriebswirtschaftlichen Erfordernisse, in deren anderer Schale der Anspruch einer hochwertigen Patientenversorgung liegt. Und weil ich Zahnerhalter bin, sage ich: Der Implantologe sollte uns die Gratwanderung nicht abnehmen.

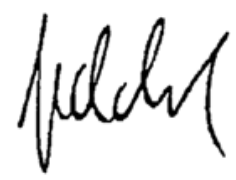

\section{Dr. J. Fedderwitz}

\section{Korrespondenzadresse}

\section{Dr. J. Fedderwitz}

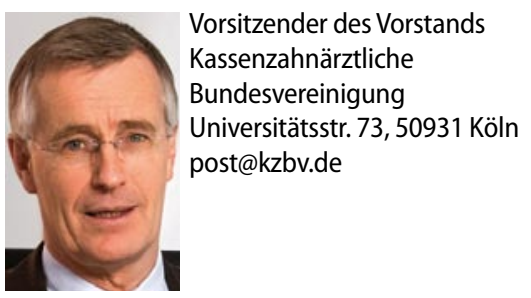

"Der MKG-Chirurg" bietet Ihnen umfassende und aktuelle Beiträge zu interessanten Themenschwerpunkten aus allen Bereichen der Mund-, Kiefer- und Gesichtschirurgie.

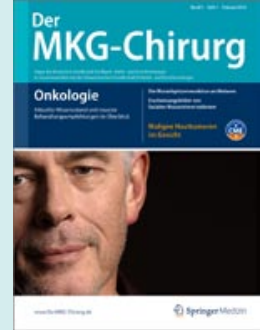

Wir haben die Jahrgänge 2009/2010 im Überblick für Sie zusammengestellt:

\section{9}

\section{- 01/09 Hautchirurgie \\ 02/09 Leitlinien und evidenzbasierte Medizin \\ - 03/09 Botulinumtoxin und Filler \\ - 04/09 Schmerztherapie}

\section{0}

- 1/10 Onkologie

- 2/10 Chirurgische Zahnerhaltung Indikationen und Alternativen

- 3/10 Qualitätsmanagement

- 4/10 Augmentationsverfahren in der Implantologie

(Änderungen vorbehalten)

Bestellen Sie einzelne Ausgaben oder abonnieren Sie die Zeitschrift zum Preis von 154,- EUR pro Jahr (zzgl. Versandkosten, Ermäßigung für Ärzte in Fortund Weiterbildung).

Unser Kundenservice steht Ihnen für Fragen und Informationen gerne zur Verfügung:

Springer Customer Service Center Haberstrasse 7, 69126 Heidelberg Tel: +49(6221)345-4303,

Fax: +49(6221)345-4229

E-Mail: Leserservice@springer.com

Eine interessante Lektüre wünscht Ihnen

Ihre Redaktion

Fachzeitschriften Medizin/Psychologie

www.DerMKG-Chirurg.de 